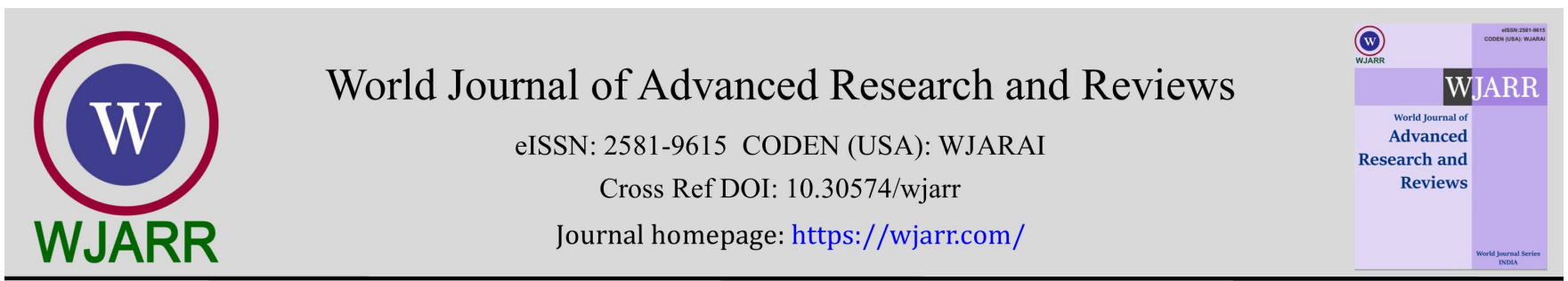

(RESEARCH ARTiClE)

\title{
Clinical picture and grouping of retinoblastoma at first presentation to a tertiary Hospital in Dar es Salaam: Implications for treatment outcomes
}

\author{
Milka Madaha Mafwiri *, Jacqueline Ngalula, Ntsilane Susan Mosenene, Celina Mhina and Anna Sanyiwa \\ Department of Ophthalmology, Muhimbili University of Health and Allied Sciences, Tanzania.
}

World Journal of Advanced Research and Reviews, 2021, 11(03), 168-177

Publication history: Received on 30 June 2021; revised on 04 August 2021; accepted on 06 August 2021

Article DOI: https://doi.org/10.30574/wjarr.2021.11.3.0363

\begin{abstract}
Objectives: To assess the clinical picture and grouping of retinoblastoma at first presentation to the oncology ward at Muhimbili National Hospital.

Methods: A cross sectional descriptive hospital based study was conducted from April to December 2018. Seventy two patients who presented for the first time to Muhimbili National Hospital (MNH) with a diagnosis of retinoblastoma were consecutively sampled and recruited in the study. Visual acuity, horizontal corneal diameter, intraocular pressure, anterior and posterior segments of the eye were assessed and each eye was classified according to the International Classification of Retinoblastoma (ICRB) group. Ultrasonography, neuroimaging and histology were performed.

Results: A total of 72 patients comprising of 90 affected eyes were recruited and analyzed: 39(54.2\%) males and $33(45.8 \%)$ females. Majority of patients were residents of the Coastal and Lake Zones. Family history of Retinoblastoma was positive in only one patient. Fifty four (75.0\%) patients had unilateral disease. Leukocoria (77.8\%) and proptosis (41.7\%) were the commonest first clinical signs noted by the family and health workers at MNH respectively. At MNH, both bilateral and unilateral cases presented with advanced disease of group E and extra ocular extension where 84(93.3\%) eyes were indicated for enucleation. The median lag time from disease onset to presentation at MNH was 4 months.
\end{abstract}

Conclusion: Leukocoria was the commonest first sign of retinoblastoma presentation at home. However, despite early presentation to primary health facilities, most patients presented to the tertiary centre of MNH late with advanced disease. Efforts to raise awareness on retinoblastoma to both health workers and the community are important for early case detection, referral, diagnosis and treatment in order to improve visual outcomes and survival rates of retinoblastoma patients.

Keywords: Retinoblastoma; Clinical Picture; ICRB Grouping; Treatment outcomes

\section{Introduction}

Retinoblastoma is the most common and important life-threatening intraocular malignancy in children which originates in immature retinal cells in one (60\%) or both eyes (40\%) [1]. It results from genetic mutations in one or more cells of the retina due to mutational inactivation of the retinoblastoma ( $R B 1)$ gene. An inactive $R B 1$ gene leads to tumor formation by allowing uncontrolled retinal cell division. The RB1 gene may be inherited leading to familial disease in $6 \%$ or may commonly develop at random leading to sporadic disease in $94 \%$ [2]. The incidence of retinoblastoma is similar in both males and females [3] and is about 1/15000 live births.

\footnotetext{
* Corresponding author: Milka Madaha Mafwiri

Department of Ophthalmology, Muhimbili University of Health and Allied Sciences, Tanzania.

Copyright (C) 2021 Author(s) retain the copyright of this article. This article is published under the terms of the Creative Commons Attribution Liscense 4.0.
} 
The median age at diagnosis of retinoblastoma is 18 months; with an average of 12 months for bilateral disease and 24 months for unilateral tumor [5] although the tumor can occasionally occur in adults [6]. Successful treatment requires early diagnosis as well as availability of modern treatment modalities to preserve the eye and save life. If left untreated the tumor fills and destroys the globe necessitating its removal by enucleation [7] and or causing death due to metastatic spread.

The International Classification of Retinoblastoma (ICRB) is based on grouping of the tumor according to clinical signs and is useful in management decision making. The five years survival rates for retinoblastoma are higher in developed countries ranging from $83 \%$ - 97\% while they are much lower at 20\% - 48\% in developing countries such as Africa and India [8]. Eye removal and low survival rates in developing countries are mainly due to delayed diagnosis and advanced presentation of retinoblastoma which is interconnected with multiple cultural and social economic factors [9]. However, in developed countries the goals of treatment have shifted towards globe and vision salvage resulting in excellent survival and vision outcomes [10]. Evidence based reports have shown that with timely screening, diagnosis, referral, treatment and follow-up delivered in a systematic way by a multidisciplinary team, $98 \%$ of children with retinoblastoma survive and their eyes and vision are preserved [11].

Previous reports in Tanzania revealed a mean lag time for presentation of about 10 months [12]. Additionally, stigma associated with removal of the eye forces parents to abandon early treatment by removal of the eye, just to present later at metastatic stage [12].

In response for the need to improve survival rates of patients with retinoblastoma, in March 2016, a National retinoblastoma program was established at $\mathrm{MNH}$ to coordinate the efforts of different organizations interested in the care of patients with retinoblastoma. In this program retinoblastoma care is performed using standardized protocol/guidelines for the country. The study was specifically conducted to identify the first clinical presentation at home and at $\mathrm{MNH}$, to group the affected eyes by the ICRB and to assess the time lag between disease onset and presentation to the tertiary centre. Study results would provide baseline information that would help the program to establish strategies for improving care of patients with Retinoblastoma.

\section{Material and methods}

\subsection{Study setting and design}

A hospital based descriptive cross-sectional study was conducted from April to December 2018, at Muhimbili National Hospital (MNH) in Dar-es-Salaam, Tanzania. Data was collected from the oncology ward where children are admitted for management of different types of cancer including retinoblastoma from the Coastal zone and the rest of the country. On average, the bed state in the oncology ward is 80 patients per day.

\subsection{Study population}

\subsubsection{Inclusion criteria}

All patients who presented to MNH for the first visit and who were confirmed to have retinoblastoma by a pediatric ophthalmologist were consecutively recruited into the study. The study population area of residency was grouped according to Tanzania geographical zones namely: the Lake, Coastal, Central, Southern, Northern and Southern highland zones.

\subsubsection{Exclusion criteria}

Patients who presented to the hospital for follow up of retinoblastoma.

\subsubsection{Data collection procedures}

After signing an informed consent, a short history was taken from the parent/caretaker to gather information on the first symptom noted, time lag between noting the first symptom to decision to seek medical care and time lag between first symptom to presentation to a health facility and management offered at that health facility. Standardized ophthalmological evaluation was done to determine the extent of disease; investigations including computerized tomography (CT scan) and blood workup were then performed. 
Patients were then examined under general anesthesia to assess the intraocular pressure, anterior segment and posterior segment of the eye; and horizontal corneal diameters. Finally each eye was grouped and recorded according to the ICRB disease group (Table 1). Delayed presentation to the tertiary centre was defined as those patients in group D, E and Extra ocular disease in whom preservation of the vision and the globe were not possible. Patients then underwent treatment according to the level of disease including enucleation, exenteration and a combination of exenteration and chemotherapy.

Table 1 International Classification of Retinoblastoma (ICRB)

\begin{tabular}{|l|l|}
\hline \multicolumn{1}{|c|}{ Group } & \multicolumn{1}{c|}{ ICRB Features } \\
\hline Group A & Small intraretinal tumors $(\leq 3 \mathrm{~mm})$ away from foveola and disc \\
\hline Group B & $\begin{array}{l}\text { Tumors }>3 \mathrm{~mm} \text {, macular location } \leq 3 \mathrm{~mm} \text { to foveola or juxtapapillary location } \leq 1.5 \\
\text { mm to disc, or with sub-retinal fluid } \leq 3 \mathrm{~mm} \text { from margin. }\end{array}$ \\
\hline Group C & $\begin{array}{l}\text { Tumor with focal sub-retinal seeds } \leq 3 \mathrm{~mm} \text { from tumour or vitreous seeds } \leq 3 \mathrm{~mm} \\
\text { from tumour or both sub-retinal and vitreous seeds } \leq 3 \text { mm from tumour. }\end{array}$ \\
\hline Group D & $\begin{array}{l}\text { Tumor with diffuse sub-retinal seeds }>3 \text { mm from tumour or vitreous seeds }>3 \mathrm{~mm} \\
\text { from tumor or both sub-retinal al and vitreous seeds }>3 \mathrm{~mm} \text { from tumour. }\end{array}$ \\
\hline Group E & $\begin{array}{l}\text { Extensive retinoblastoma occupying }>50 \% \text { of the globe with or without neovascular } \\
\text { glaucoma, opaque media from hemorrhage in anterior chamber, vitreous or sub- } \\
\text { retinal space. Extension of tumor to post lamina optic nerve, CHOROID> 2 mm, } \\
\text { sclera and anterior chamber. }\end{array}$ \\
\hline Extra ocular/Orbital & Involvement of extraocular muscles and or orbit \\
\hline Unclassified & $\begin{array}{l}\text { Destructed anterior segment or dense media opacity obscuring fundus view / } \\
\text { patients who came already enucleated. }\end{array}$ \\
\hline
\end{tabular}

\subsection{Data analysis}

Data were analyzed with the help of Statistical Package for Social Sciences (SPSS) software version 20. Univariate analysis was done to assess characteristics of the study population. Bivariate analysis using Chi-square or Fisher's exact tests was used to assess the association between tumor laterality and patient age at disease onset. Any association was considered significant at $95 \%$ confidence interval.

\subsection{Ethical Consideration}

Approval to conduct the study was granted by the Senate Research and Publication Committee of MUHAS. Permission to conduct the study was given by the Director General of MNH. Parents and care takers consented for their children to take part in the study as well as to undergo examination under general anesthesia.

\section{Results}

A total of 312 children were diagnosed with different cancers in the oncology ward during the study period. Seventy two $(23 \%)$ children with retinoblastoma met the inclusion criteria and were all included in the analysis.

Most (63\%) patients were aged between 1 to 48 months (63\%), males (54.1\%), residents of Coastal and Lake Zones $(61 \%)$, with unilateral disease (75\%), and without family history of retinoblastoma or consanguinity (Table 2). Patients with bilateral disease were 16.30 months younger than those with unilateral.

The commonest first clinical presentation noted by the family at home was leukocoria while proptosis associated with leukoria was the commonest presentation at MNH. (Table 3).

All (100\%) eyes with unilateral disease and $83.3 \%$ eyes with bilateral disease presented with advanced disease group where vision and globe salvage was not possible (Table 4). 
Table 2 Clinical and demographic characteristics of the study population stratified by sex. N=72

\begin{tabular}{|c|c|c|c|}
\hline \multirow{2}{*}{ Characteristic } & Male $n=39$ & Female $n=33$ & Total $n=72$ \\
\hline & N (\%) & N (\%) & N (\%) \\
\hline \multicolumn{4}{|l|}{ Age group (months) } \\
\hline $0-24$ & $18(46.2)$ & $16(48.5)$ & $34(47.2)$ \\
\hline $25-48$ & $16(41.0)$ & $13(39.4)$ & $29(40.3)$ \\
\hline$>48$ & $5(12.8)$ & $4(12.1)$ & $9(12.5)$ \\
\hline Median age (IQR) & $30(16-45)$ & $26(20-36)$ & $28(17-42)$ \\
\hline \multicolumn{4}{|l|}{ Patient address } \\
\hline Lake zone & $12(30.8)$ & 9 (27.3) & $21(29.2)$ \\
\hline Coastal zone & $10(25.6)$ & $13(39.4)$ & $23(31.9)$ \\
\hline Southern zone & 7 (17.9) & $2(6.0)$ & $9(12.5)$ \\
\hline Southern highlands zone & $4(10.3)$ & $4(12.1)$ & $8(11.1)$ \\
\hline Central zone & $6(15.4)$ & $5(15.2)$ & $11(15.3)$ \\
\hline \multicolumn{4}{|l|}{ Family History of RB } \\
\hline Yes & $1(2.6)$ & $0(0.0)$ & $1(1.4)$ \\
\hline No & 38 (97.4) & $33(100.0)$ & $71(98.6)$ \\
\hline \multicolumn{4}{|l|}{ Consanguinity history } \\
\hline Yes & $1(2.6)$ & $0(0.0)$ & $1(1.4)$ \\
\hline No & $38(97.4)$ & $33(100.0)$ & $71(98.6)$ \\
\hline \multicolumn{4}{|l|}{ Tumor Laterality } \\
\hline Unilateral & $29(74.4)$ & $25(75.8)$ & $54(75.0)$ \\
\hline Bilateral & $10(25.6)$ & $8(24.2)$ & $18(25.0)$ \\
\hline
\end{tabular}

Table 3 Clinical signs of patients as first seen at home and $\mathrm{MNH}$

\begin{tabular}{|l|c|c|c|}
\hline \multirow{2}{*}{ Clinical sign } & Male n = 39 & Female n = 33 & Total n = 72 \\
\cline { 2 - 4 } & N (\%) & N (\%) & N (\%) \\
\hline First sign noted by the family & \multicolumn{2}{|c|}{} \\
\hline Leucokoria & $28(71.7)$ & $28(84.9)$ & $56(77.8)$ \\
\hline Strabismus & $4(10.3)$ & $1(3.0)$ & $5(6.9)$ \\
\hline Red eye & $4(10.3)$ & $3(9.1)$ & $7(9.7)$ \\
\hline Proptosis & $1(2.6)$ & $1(3.0)$ & $2(2.8)$ \\
\hline Others & $2(5.1)$ & $0(0.0)$ & $2(2.8)$ \\
\hline Sign noted at MNH & & & \\
\hline Leucokoria & $19(48.7)$ & $10(30.3)$ & $29(40.2)$ \\
\hline Strabismus & $2(5.1)$ & $0(0.0)$ & $2(2.8)$ \\
\hline Red eye & $1(2.6)$ & $2(6.0)$ & $3(4.2)$ \\
\hline Proptosis & $12(30.8)$ & $18(54.6)$ & $30(41.7)$ \\
\hline Others & $5(12.8)$ & $3(9.1)$ & $8(11.1)$ \\
\hline
\end{tabular}


Table 4 ICRB grouping of affected eyes in unilateral and bilateral retinoblastoma

\begin{tabular}{|l|c|c|}
\hline \multicolumn{3}{|c|}{ Unilateral Rb n=54 eyes } \\
\hline Group & No & \% \\
\hline Group A & 0 & 0.0 \\
\hline Group B & 0 & 0.0 \\
\hline Group C & 0 & 0.0 \\
\hline Group D & 6 & 11.1 \\
\hline Group E & 13 & 24.1 \\
\hline Extra ocular/orbital & 21 & 38.9 \\
\hline Unclassified & 14 & 25.9 \\
\hline Total & 54 & 100 \\
\hline Bilateral Rb n=36 eyes & & \\
\hline Group A & 0 & 0.0 \\
\hline Group B & 1 & 2.8 \\
\hline Group C & 5 & 13.9 \\
\hline Group D & 7 & 19.5 \\
\hline Group E & 12 & 33.3 \\
\hline Extra ocular/orbital & 8 & 22.2 \\
\hline Unclassified & 3 & 8.3 \\
\hline Total & 36 & 100 \\
\hline
\end{tabular}

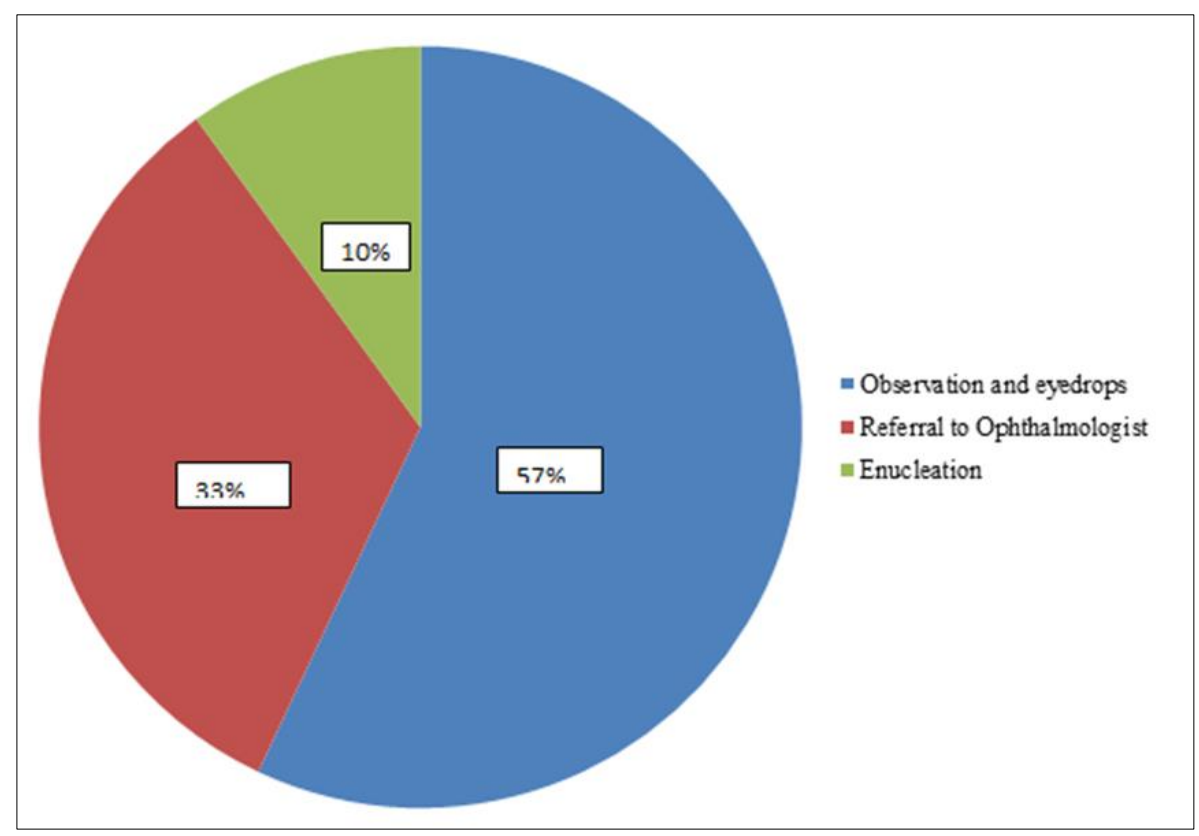

Figure 1 Treatment modalities provided at the primary health facilities 
Most (84\%) parents/caregivers decided to seek medical care within 6 months, where $48(66.7 \%)$ visited the health facility within 6 months with a median lag time of 4 months from disease onset. Dispensaries and health centers were the first health facility contacted by 48 (66.7\%.) patients. (Table 5).

Table 5 Characteristics of study participants lag time stratified by sex

\begin{tabular}{|l|c|c|c|}
\hline \multirow{2}{*}{ Characteristic } & Male n= 39 & Female n = 33 & Total n = 72 \\
\cline { 2 - 4 } & N (\%) & N (\%) & N (\%) \\
\hline Age of disease onset in months & $24(61.5)$ & $20(60.6)$ & $44(61.1)$ \\
\hline $0-24$ & $12(30.8)$ & $10(30.3)$ & $22(30.6)$ \\
\hline $25-48$ & $3(7.7)$ & $3(9.1)$ & $6(8.3)$ \\
\hline$>48$ & $35(89.7)$ & $26(78.8)$ & $61(84.7)$ \\
\hline Time taken to decide to seek medical care (months) \\
\hline$<6$ & $4(10.3)$ & $7(21.2)$ & $11(15.3)$ \\
\hline$>6$ months & $7(17.9)$ & $10(30.3)$ & $17(23.6)$ \\
\hline First visit facility level & $14(35.9)$ & $12(36.4)$ & $26(36.1)$ \\
\hline Dispensary & $15(38.5)$ & $9(27.3)$ & $24(33.3)$ \\
\hline Health centre & $2(5.1)$ & $1(3.0)$ & $3(4.2)$ \\
\hline Regional hospital & $1(2.6)$ & $1(3.0)$ & $2(2.8)$ \\
\hline Referral hospital & & \\
\hline Other places & $30(76.9)$ & $18(54.6)$ & $48(66.7)$ \\
\hline Lag time from onset to first health facility (months) & $5(12.8)$ & $12(36.4)$ & $17(23.6)$ \\
\hline $0-6$ & $4(10.3)$ & $3(9.0)$ & $7(9.7)$ \\
\hline $7-12$ & $2(1-6)$ & $6(2-10)$ & $4(1.5-8)$ \\
\hline$>12$ &
\end{tabular}

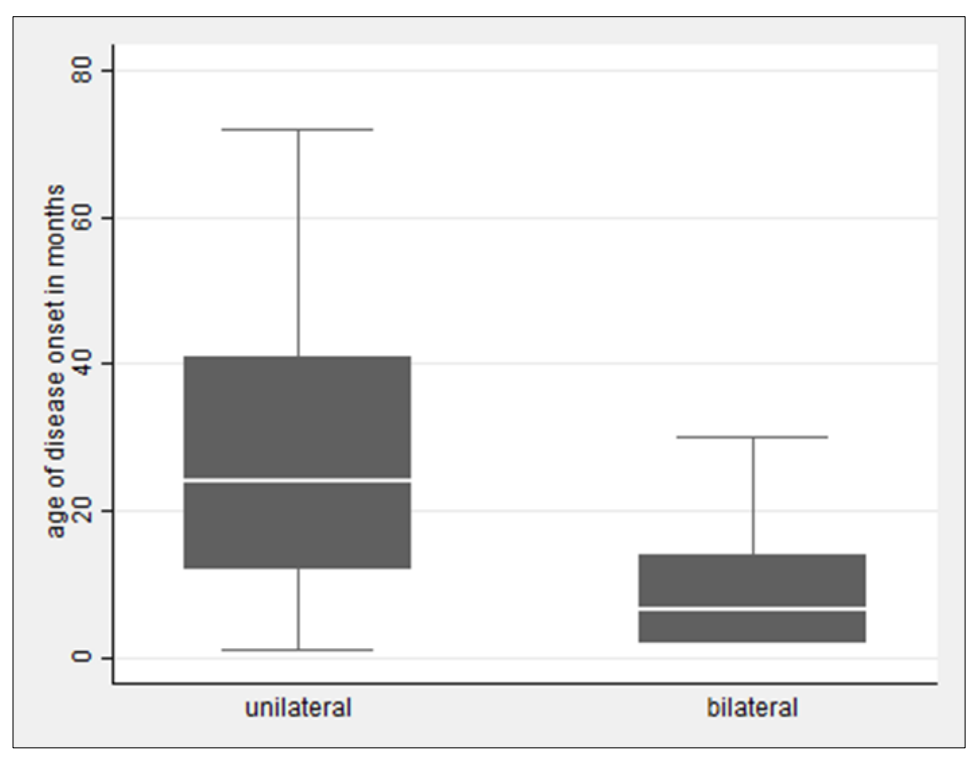

Figure 2 Box plot of median age of onset by tumor laterality 
Observation and eye drops were the most common (57\%) type of treatment given at peripheral health units at first presentation. Immediate referral to ophthalmologist was done to about a third of the children (Figure 1).

Patients with bilateral tumor were 16.30 months younger than those with unilateral tumor and the difference was statistically significant $(\mathrm{p}<0.001)$ (Figure 2 ).

\section{Discussion}

Results of this study show that almost a quarter of children admitted in the Oncology ward at MNH suffer from retinoblastoma. This is a reflection of collection of patients at the only well-established pediatric oncology centre in the country. However, this high proportion might be a true reflection of a high prevalence of retinoblastoma in Africa as it has been shown in literature [13]. There is evidence that the prevalence of retinoblastoma is not distributed equally worldwide, appearing to be higher in Africa, India, and among children of Native American descent [13]. The source of this variation is not known. However, the fact that even in industrialized countries an increased incidence of retinoblastoma is associated with poverty and low levels of maternal education, suggests an environmental role [14]. Although this study did not evaluate the social economic status and level of education of parents/care takers most of them came from poor rural areas. We propose a study to explore whether there is a relationship between social economic status and retinoblastoma to be done in the future.

The study found that most patients came from the Coastal and Lake Zones. MNH is within the Coastal zone making it easier for patients to access services. On the other hand, the Lake zone is more than $1000 \mathrm{KM}$ from MNH. Although there is a tertiary hospital in Mwanza (Bugando) which is located in the Lake Zone, during the study period, there were no Oncology services to cater for patients from this area. It is also likely that these two zones are the areas where retinoblastoma is more prevalent. Establishment of oncology services at Bugando tertiary referral hospital with a Retinoblastoma management program alongside a Retinoblastoma awareness campaigns cannot be over emphasized. No patient came from Northern highland zone probably due to the availability of Retinoblastoma services at the Kilimanjaro Christian Medical Centre.

In this study there were slightly more boys than girls with retinoblastoma. This finding was similar to other studies which showed slightly male predominance $[12,14]$. However, a study done by Balmer A et al [15] suggested no difference in sex. Another study done by E Broaddus et al [16] suggested mild female preponderance; the difference however was not significant. Finding more males than females in this study may be related to the fact that our social and cultural situations in the African societies favor a male child more in education and health needs compared to a female child.

Our study findings are in line with evidence from literature that retinoblastoma commonly affects children below the age of 5 years [1] where most are unilateral [17].

In this study only one (1.4\%) patient with bilateral retinoblastoma had a strong positive family history of having 2 brothers who had bilateral retinoblastoma and only 1(1.4\%) patient came from a family and area where consanguinity is practiced, the parents were cousins. Apart from the Coast area of the Indian Ocean and especially Zanzibar, most parts of Tanzania mainland do not practice consanguinity. These findings are similar to a study done in Malaysia where history of consanguinity was positive in only one patient [17].

The reported median age at presentation for Retinoblastoma is 18 months with an average of 12 months for bilateral disease and 24 months for unilateral tumors [18]; while for this report the median age for presentation was 28 months with an average of 6.5 months for bilateral cases an 24 months for unilateral cases. Those with bilateral disease were 16.30 months younger than those with unilateral disease and the difference was statistically significant $(\mathrm{p}<0.001)$.

Available evidence shows that leukocoria is the most common presenting feature of retinoblastoma, followed by strabismus, painful blind eye and loss of vision [7]. The findings are similar to those reported by Abramson et al [19]. In our study, although majority reported leukocoria as a first sign during disease onset, both bilateral and unilateral cases had advanced disease at the time of presentation at MNH. According to ICRB, 84 out of 90 eyes with Retinoblastoma were in groups D, E, extraocular and unclassified and the outcome for this classification was enucleation while only 6 eyes were for organ salvage. All 84 eyes were enucleated due to advanced disease stage. Removal of eyes contributes significantly to childhood blindness and leads to impaired quality of life to survivors and their families. Three patients had bilateral enucleations. 
Our results show a longer period between onset of disease and presentation to the tertiary centre where most children with unilateral disease presented with advanced disease than those with unilateral disease. A previous report from Tanzania revealed that the time delay between noting the first sign of retinoblastoma to the time of presentation to an ophthalmologist for expertise medical help was about 10 months [12] whereas an overall median lag time of 4 months was observed in this study. The decrease in lag time from 10 months to 4 months may be attributed to the awareness campaign done along with the establishment of retinoblastoma services at MNH which has improved access for care. Efforts to create more awareness in the community and among primary health workers are necessary to ensure early identification and referral to tertiary centres for management. The inclusion of an eye health module in the training program for the Integrated Management of Childhood Illnesses in 2018 in Tanzania allows primary health workers to improve their knowledge and skills in the diagnosis and referral of children with eye diseases including retinoblastoma [20]

Results of our study show that although most parents had noted leukocoria (77.8\%), a smaller proportion (66.7\%) decided to seek medical care within 6 months and eventually most patients presented at the tertiary centre late, with advanced disease. Presentation at advanced stage has poor prognostic factors and contributes to the high mortality in the range of 50-70\% in developing countries [12.] Seven of our patients had central nervous system involvement at first presentation and they could not be treated, and they died during the study period.

Late presentation for treatment in African countries has been documented in the literature [17] The reasons include lack of awareness about the causation of disease and how serious it is among the parents and the general population. This deters parents to seek for treatment immediately after noticing leukocoria. Another delay happens at the first point health facility where due to inadequate knowledge and skills, primary health care providers misdiagnose and unnecessarily delay to refer patients with Retinoblastoma to specialized centers for appropriate treatment [21] Most $(84.7 \%)$ of our patients sought medical care within 6 months of disease onset from a nearby primary health facility. In Tanzania, these primary health facilities are staffed by primary health care providers including medical assistants, enrolled nurses, and medical attendants with inadequate knowledge and skills about diagnosis and management of retinoblastoma or other eye conditions. Additionally, the leucokoria from retinoblastoma is not seen constantly. So when parents report this sign but the health worker does not see it, or the child appears to be feeding and playing normally, primary health workers conclude that the eye is healthy and consequently observe the child on eye drops. More than half (57\%) of our patients were observed while only $10 \%$ were referred.

Late presentation to the tertiary centre is also contributed by the structure of the health referral system in Tanzania which follows a hierarchy system from primary to tertiary level. The system does not allow a direct referral to a tertiary hospital. Patients with retinoblastoma have to pass through the dispensary, health Centre, district and finally regional referral hospital before they reach the tertiary MNH. A previous study in Dar-es-Salaam among primary health facility workers pointed out a long referral chain as a barrier towards timely referral of children with eye conditions including congenital cataract and retinoblastoma to appropriate health facilities [21]. To improve care for patients with retinoblastoma, it is necessary to establish strategies to shorten this referral pathway.

\section{Conclusion}

About a quarter of patients admitted in the oncology ward at MNH in Dar-es-Salaam suffer from retinoblastoma. Most affected children were less than 5 years and had unilateral disease. The first clinical sign was leukocoria and although most parents sought medical care within 6 months of onset of disease, they presented late to the tertiary hospital of MNH with advanced disease within ICRB groups E and extra ocular/ orbital disease where globe and vision salvage were not possible.

\section{Recommendation}

Raising awareness on Retinoblastoma to both primary health workers and the public at large on the importance of early screening, diagnosis and referral of affected patients is recommended. A further long term study is proposed on long term outcome and social economic factors of the patients who are managed at $\mathrm{MNH}$.

\section{Study Limitations}

We cannot generalize our findings to the rest of the country, but only to tertiary centres. 


\section{Compliance with ethical standards}

\section{Acknowledgments}

We are thankful to the Ministry of Education for funding the study.

\section{Disclosure of conflict of interest}

The authors declare no conflict of interests. MMM designed the study, collected data, and wrote the manuscript. JN designed the study, collected data, performed data analysis and prepared the initial manuscript. SNM participated in the study design. CM and reviewed the manuscript. AJS participated in the study design, data collection and report writing.

\section{Statement of ethical approval}

Approval to conduct the study was granted by the Senate Research and Publication Committee of MUHAS. Permission to conduct the study was given by the Director General of MNH.

\section{Statement of informed consent}

The study commenced only after parents had signed a written informed consent to participate in the study as well as to undergo examination under anesthesia.

\section{References}

[1] Abramson DH. Retinoblastoma: diagnosis, treatment and implications. Pediatr Ann. 1990; 19: 387.

[2] Shields CL, Shields J. Diagnosis and management of retinoblastoma. Cancer Control. 2004; 11(5): 317-27.

[3] Sabyasachi Sengupta, Utsab Pan, Vikas Khetan. Adult onset retinoblastoma. Indian J Ophthalmol. 2016; 64(7): 485-91.

[4] Rodriguez-Galindo C, Orbach DB, Vanderveen D. Retinoblastoma. Pediatr Clin North Am. 2015; 62: 201-223. 2015.

[5] Goddard G, Kingston JE, Hungerford JL. Delay in diagnosis of retinoblastoma: risk factors and treatment outcome. Br J Ophthalmol. 1999; 83(12): 1320-3.

[6] Shields Carol L, Shields JA. Retinoblastoma management: advances in enucleation, intravenous chemoreduction, and intra-arterial chemotherapy. Curr Opin Ophthalmol. 2010; 21(3): 203-12.

[7] Malkani I, Warrier RP, Yu LC, Ode DL. Retinoblastoma: A review. Indian J Pediatr. 1993; 60(2): 227-236.

[8] MacCarthy A, Birch JM, Draper GJ, Hungerford JL, Kingston J E, Krol MEl, Stiller CA, Vincent TJ. Retinoblastoma: treatment and survival in Great Britain. Br J Ophthalmol. 2009; 93(1): 38-9.

[9] Kushner BH, LaQuaglia MP, Wollner N, Meyers PA, Lindsley KL, Ghavimi F, Merchant TE, Boulad F, Cheung N K, Bonilla MA, Crouch G, Kelleher JF Jr, Steinherz P G, Gerald WL. Desmoplastic small round-cell tumor: Prolonged progression-free survival with aggressive multimodality therapy. J Clin Oncol. 1996; 14(5): 1526-31.

[10] Jhagta HS, Jain P, Gupta M et al. Retinoblastoma : A Curse to Childhood. Indian J Clin Pract. 2013; 24(2): 175-8.

[11] Epee E, Moukouri E, Koki G, Pondy A, Mbassi K. Clinical Features and Prognosis of Retinoblastoma at the University Teaching Hospital of Yaounde - Cameroon. Heal Sci Dis. 2014; 15(3): 1-6.

[12] Bowman RJ, Mafwiri M, Luthert P, Luande J. Outcome of retinoblastoma in east Africa. Pediatr Blood Cancer. 2008; 50(1): 160-2.

[13] Wilson O Akhiwu, Alex P Igbe. Retinoblastoma: An Update on Clinical, Genetic Counseling(Ed.). 2012; ISBN: $978-$ 953-51-0435-3.

[14] Nyamori JM, Kimani K, Njuguna MW, Dimaras H. The Incidence and Distribution of Retinoblastoma in Kenya. Br J Ophthalmol. 2012; (1): 4-5. 
[15] Balmer A, Zografos L, Munier F. Diagnosis and current management of retinoblastoma. NCBI Oncogene. 2006; 25(38): 5341-9.

[16] E Broaddus, A Topham, Singh AD. Incidence of retinoblastoma in the USA: Br J Ophthalmol. 2009; 93(1): 21-3.

[17] Subramaniam S, Rahmat J, Rahman NA, Ramasamy S, Bhoo-Pathy N, Pin GP, Alagaratnam J. Presentation of retinoblastoma patients in Malaysia. Asian Pac J Cancer Prev. 2014; 15(18): 7863-7.

[18] Singh SK, Das D, Bhattacharjee H, Biswas J, Kuri G, Bhattacharjee K, Deka H, Deka AC. A rare case of adult onset retinoblastoma. Oman J Ophthalmol. 2011; 4(1): 25-7.

[19] Abramson DH. The diagnosis of retinoblastoma. Bull N Y Acad Med. 1988; 64(4): 283-317.

[20] Malik ANJ, Mafwiri M, Gilbert C, Kim MJ, Schellenberg J. Integrating eye health training into the primary child healthcare programme in Tanzania: a pre-training and post-training study. BMJ Paediatr Open. 2020; 4(1): e 000629.

[21] Mafwiri MM, Kisenge R, Gilbert CE. A pilot study to evaluate incorporating eye care for children into reproductive and child health services in Dar-es-Salaam, Tanzania: a historical comparison study. BMC Nurs. 2 Jun 2014; 13: 15 . 